\title{
An Approximation of Minimum Initial Capital of Investment Discrete Time Surplus Process with Weibull Distribution in a Reinsurance Company
}

\author{
Soontorn Boonta ${ }^{1}{ }^{1}$ and Somchit Boonthiem ${ }^{2}$ \\ ${ }^{1}$ Department of General Science, Faculty of Science and Engineering, Kasetsart University, Chalermphrakiat Sakon Nakhon Province \\ Campus, Sakon Nakhon 47000, Thailand \\ ${ }^{2}$ Mathematics and Statistics Program, Faculty of Science, Sakon Nakhon Rajabhat University, Sakon Nakhon 47000, Thailand
}

Correspondence should be addressed to Soontorn Boonta; soontorn.bo@ku.th

Received 31 January 2019; Accepted 23 May 2019; Published 11 June 2019

Academic Editor: Ying Hu

Copyright (c) 2019 Soontorn Boonta and Somchit Boonthiem. This is an open access article distributed under the Creative Commons Attribution License, which permits unrestricted use, distribution, and reproduction in any medium, provided the original work is properly cited.

\begin{abstract}
Catastrophe is a loss that has a low probability of occurring but can lead to high-cost claims. This paper uses the data of fire accidents from a reinsurance company in Thailand for an experiment. Our study is in two parts. First, we approximate the parameters of a Weibull distribution. We compare the parameter estimation using a direct search method with other frequently used methods, such as the least squares method, the maximum likelihood estimation, and the method of moments. The results show that the direct search method approximates the parameters more precisely than other frequently used methods (to four-digit accuracy). Second, we approximate the minimum initial capital (MIC) a reinsurance company has to hold under a given ruin probability (insolvency probability) by using parameters from the first part. Finally, we show MIC with varying the premium rate.
\end{abstract}

\section{Introduction}

The risks of an insurance company can be assessed based on disasters of varying severity. The insurance company evaluates its risks in order to maintain consistency. Insolvency cannot occur if the company knows how to manage the risk process. For instance, if an insurance company does not have sufficient initial capital to pay some claims, then the company can share some of the risks by transferring them to reinsurance. Parameter estimation is an important method to construct a risk model in an insurance business. The wellknown methods are the least squares method, the maximum likelihood estimation, and the method of moments. This research is interested in the estimation of the parameters of a Weibull distribution which are represented by fire accident data. Many authors have studied the different aspects of Weibull parameters. Bergman [1] and Sullivan and Lauzon [2] proposed four probability estimators which were frequently applied in the least squares method. Based on a Monte Carlo simulation, Khalili and Kromp [3] and Trustrum and
Jayatilaka [4] compared an estimation of Weibull parameters by using the least squares method, the maximum likelihood estimation, and the method of moments. Boonta et al. [5] proposed a direct search technique to estimate the parameters of Weibull distribution. They compared the Chisquared value of the direct search technique to the least squares method, the maximum likelihood estimation, and the method of moments. The results showed that the direct search technique gave a more precise estimation than the least squares method, the maximum likelihood estimation, and the method of moments.

In this paper, we start by introducing the surplus of nonlife insurance. The surplus can be described as

$$
\text { Surplus }=\text { Initial capital }+ \text { Income }- \text { Outflow } \text {. }
$$

Lundberg [6] was the first actuary who considered the surplus process of nonlife insurance under three assumptions in his model.

(1) Claims happening at times $T_{i}$ satisfying $0 \leq T_{1} \leq T_{2} \leq$ ... are called claim arrivals or claim times. 
(2) The $i$-th claim arriving at time $T_{i}$ causes the claim size or claim severity $Y_{i}$. The sequence of claim sizes $\left\{Y_{i}, i \in \mathbb{N}\right\}$ constitutes an independent and identically distributed (i.i.d.) sequence of nonnegative random variables.

(3) The claim severity process $\left\{Y_{i}, i \in \mathbb{N}\right\}$ and the claim arrival process $\left\{T_{i}, i \in \mathbb{N}\right\}$ are mutually independent.

Next, we define the claim number process

$$
N(t)=\#\left\{i \geq 1: T_{i} \leq t\right\}, \quad t \geq 0 .
$$

Thus, $N(t)$ is the number of claims in $[0, t]$.

Next, we denote $\left\{Y_{n}, n \in \mathbb{N}\right\}$ as the i.i.d. process of the claims, $u$ as the initial capital, and $c$ as the premium rate for one unit of time. There are many research studies of ruin probability in terms of initial capital $u$ (see in Pavlova and Willmot [7], Dickson [8] Li [9, 10], and Rongming and Haifeng [11]). Chan and Zhang [12] have studied a discrete time surplus process such as claim time $T_{n}=n$ (claims which occur every day). They proposed recursive and explicit formulae of the ruin probabilities which are in the form

$$
U_{n}=u+c n-\sum_{k=1}^{n} Y_{k}, \quad U_{0}=u, n \in \mathbb{N}
$$

Sattayatham et al. [13] generalized the results of Chan and Zhang for claim times which do not occur every day. Their model is of the form

$$
U_{n}=u+c T_{n}-\sum_{k=1}^{n} Y_{k}, \quad U_{0}=u, n \in \mathbb{N}
$$

This can be rewritten as

$$
U_{n}=U_{n-1}+c Z_{n}-Y_{n}, \quad U_{0}=u
$$

where $\left\{Z_{n}, n \in \mathbb{N}\right\}$ is the inter arrival time process, assuming i.i.d. such that $Z_{1} \sim \operatorname{Poisson}(\lambda)$. Since the formula of ruin probability is difficult to find explicitly, they proposed the ruin probability in the recursive form:

$$
\Phi_{N}(u)=\Phi_{1}(u)+\int_{-\infty}^{u+c} \Phi_{N}(u+c-y) d F_{Y_{1}}(y),
$$

where $\Phi_{N}(u)=P\left(\left\{U_{i}<0\right.\right.$ for some $\left.i \in\{1,2, \ldots, N\}\right\}$ | $\left.U_{0}=u\right)$. Chengguo Weng et al. [14] studied a model of ruin probability by adding some investments as

$$
U_{n}=U_{n-1}(1+r)+c-Y_{n}, \quad n=1,2,3, \ldots,
$$

where $r$ is the constant interest rate for a period of time $n$. They considered $\left\{Y_{n}, n \in \mathbb{N}\right\}$ as a sequence of dependent individuals that have a regular variation distribution and zero index of upper tail dependence. They also established some asymptotic results for both finite ruin probability and ultimate ruin probability.

Reinsurance and investment are a normal activity of insurance companies because reinsurance can reduce the risk (ruin probability) arising from claims, and the investment can make more profit for the company. The process can be controlled by reinsurance, i.e., by choosing the retention level (or risk exposure) $b \in[\underline{b}, \bar{b}]$ of a reinsurance for one period. The (measurable) function $h(b, y)$ specifies the part of the claim $y$ paid by the insurer. Then $h(b, y)$ depends on the retention level $b$ (fixed in the risk sharing contract) at the beginning of the respective period where $0 \leq h(b, y) \leq y$. Hence $y-h(b, y)$ is the part paid by the reinsurer. It is natural to assume that $h(b, y)$ is increasing in $b$.

In the case of an excess of loss reinsurance we have

$$
h(b, y)=\min (b, y)
$$

with retention level $0 \leq \underline{b} \leq b \leq \bar{b}=\infty$.

In case of a proportional reinsurance we have

$$
h(b, y)=b \cdot y
$$

with retention level $0 \leq \underline{b} \leq b \leq \bar{b}=1$.

Therefore, the retention level $\bar{b}$ stands for the control action "no reinsurance" which explains the property " $c=$ $c(\bar{b})$ ". The smallest retention level $\underline{b}$ may be chosen in such a way that the condition $0 \leq c(b) \leq c=c(\bar{b})$ is satisfied. Then $c(b)$ may be calculated according to the expected value principle with safety loading $\theta$ of the reinsurer:

$$
c(b)=c-(1+\theta) \cdot \frac{E[Y-h(b, Y)]}{E[Z]} .
$$

Recently, Luesamai and Chongcharoen [15] expanded the risk model by adding proportional reinsurance and investment. Insurers can invest in the bond and stock markets, and they assume that the interest rates of the bonds have a finite number of possible values and follow a time-homogenous Markov chain. Moreover, they assume that the controlling reinsurance and stock investment values in each time period are constant values. For every time period unit $(n-1, n]$, the risk model is formulated as

$$
U_{n}=U_{n-1}\left(1+I_{n}\right)+\alpha_{n} W_{n}+c\left(b_{n}\right)-h\left(b_{n}, Y_{n}\right)
$$

where $h\left(b_{n}, Y_{n}\right)$ is computed by a proportional reinsurance function, and the sequences $\left\{I_{n}\right\},\left\{W_{n}\right\}$, and $\left\{Y_{n}\right\}$ are mutually independent. The interest rate $I_{n}$ is assumed to follow a time-homogeneous Markov chain (for $i=1,2,3, \ldots$ ). $W_{n}$ is the gross return and is assumed to be a sequence of i.i.d. nonnegative random variables. $\alpha_{n} \geq 0$ is the amount of stock investment. The results of the study led to the proposal of two upper bounds of ruin probability under a discrete time risk model for reinsurance by generalizing the classical model in terms of two controlling factors: proportional reinsurance and investment.

In this research, we study a risk model of reinsurance by adding investment (buying bonds or fixed accounts). We present two parts consisting of an approximation of the parameters of Weibull distribution and the calculation of the minimum initial capital of investment discrete time surplus process with Weibull distribution. The first part is the estimation of the Weibull parameters using the direct search technique, the least squares method, the maximum likelihood estimation, and the method of moments. The 
selected method gives the minimum KS statistic value (to four-digit accuracy). The second part is a simulation to calculate the ruin probability of the surplus process under the condition that a reinsurance company can invest in risk-free assets (bonds or fixed accounts). The surplus process is of the form

$$
\begin{aligned}
& U_{n}=U_{n-1}(1+r)+c Z_{n}-Y_{n}, \\
& \quad n=1,2,3, \ldots, U_{0}=u,
\end{aligned}
$$

where $\left\{Y_{n}, n \in \mathbb{N}\right\}$ is the i.i.d. process of the claims, $u$ is the initial capital, and $r$ is the daily interest rate which can calculated by $r=\left(1+r_{0}\right)^{1 / 365}-1, r_{0}=2 \%$ per annum. $c$ is the premium rate for one unit of time which can be computed by $c=(1+\theta)\left(E Y_{1} / E Z_{1}\right)$ where $\theta$ is a safety loading and $\left\{Z_{n}, n \in\right.$ $\mathbb{N}\}$ is the inter arrival time process, and assuming i.i.d. such that $Z_{1} \sim \operatorname{Poisson}(\lambda)$. We approximate the minimum initial capital (MIC) an insurance company has to hold under a given ruin probability (insolvency probability) by using parameters from the first part.

\section{Materials and Methods}

2.1. Weibull Distribution. The classical Weibull distribution is useful for reliability engineering. Moreover, it can be extended to the various families of probability distributions which deal with the estimation of model parameters by maximum likelihood and it can also be used to illustrate the potentiality of the extended family with two applications to real data [16]. Furthermore, a nonclassical Weibull distribution can be used to estimate the statistical characteristics in a cellular automaton such that a cell's yield stress is assumed to be a Weibull distribution [17].

Normally, claims that occur infrequently but have high costs will be called catastrophe losses. For example, a fire accident is a type catastrophe loss. Furthermore, a Weibull distribution that shape parameter being less than one and scale parameter being greater than zero is also an example of catastrophe loss. The probability density function of three parameters of a Weibull distribution is of the form

$$
f(y ; \alpha, \beta, \gamma)=\frac{\alpha}{\beta}\left(\frac{y-\gamma}{\beta}\right)^{\alpha-1} e^{-((y-\gamma) / \beta)^{\alpha}}
$$

and the cumulative distribution function is of the form

$$
F(y ; \alpha, \beta, \gamma)=1-e^{-((y-\gamma) / \beta)^{\alpha}}
$$

for all $y \geq \gamma$, where $\alpha$ is a positive shape parameter, $\beta$ is a positive scale parameter, and $\gamma$ is a positive location parameter, respectively.

In our work, the costs of all claims $y$ are greater than twenty million Baht. We establish $\gamma=20$ and $x=y-\gamma$. Thus we revise (13) and (14) as (15) and (16)

$$
f(x ; \alpha, \beta)=\frac{\alpha}{\beta}\left(\frac{x}{\beta}\right)^{\alpha-1} e^{-(x / \beta)^{\alpha}},
$$

and

$$
F(x ; \alpha, \beta)=1-\exp \left(-\left(\frac{x}{\beta}\right)^{\alpha}\right)
$$

\subsection{Weibull Parameter Estimation}

2.2.1. Least Squares Method. Bergman, Sullivan, and Lauzon proposed the probability estimator $F_{j}$ for the $j$ th ranked $y_{j}$ and $n$ is the sample size as shown in the following equations:

$$
\begin{aligned}
& F_{j}=\frac{j-0.375}{n+0.25} \\
& F_{j}=\frac{j-0.3}{n+0.4} \\
& F_{j}=\frac{j}{n+1} \\
& F_{j}=\frac{j-0.5}{n}
\end{aligned}
$$

We take the natural logarithm to (16),

$$
\ln \ln \left[\frac{1}{1-F}\right]=\alpha \ln x-\alpha \ln \beta
$$

Then we set $u=\ln \ln [1 /(1-F)], m=\alpha, v=\ln x$ and $b=$ $-\alpha \ln \beta$. Therefore, (18) is the linear equation which is of the form $u=m v+b$.

2.2.2. Maximum Likelihood Estimation. Let $\left\{x_{1}, x_{2}, \ldots, x_{n}\right\}$ be the samples from a Weibull distribution. A log-likelihood function $\ln L$ is defined by

$$
\ln L\left(x_{1}, x_{2}, \ldots, x_{n} ; \alpha, \beta\right)=\sum_{i=1}^{n} \ln \frac{\alpha}{\beta}\left(\frac{x_{i}}{\beta}\right)^{\alpha-1} e^{-\left(x_{i} / \beta\right)^{\alpha}} .
$$

By partial derivative the log-likelihood function $\partial \ln L / \partial \beta, \partial \ln L / \partial \alpha$, and setting to zero, we have

$$
\begin{aligned}
\frac{\partial \ln L}{\partial \beta} & =-\frac{n}{\beta}+\frac{1}{\beta^{\alpha+1}} \sum_{i=1}^{n}\left(x_{i}\right)^{\alpha}=0, \\
\frac{\partial \ln L}{\partial \alpha} & =\frac{n}{\alpha}-n \ln \beta+\sum_{i=1}^{n} \ln x_{i}-\sum_{i=1}^{n}\left(\frac{x_{i}}{\beta}\right)^{\alpha} \ln \left(\frac{x_{i}}{\beta}\right) \\
& =0 .
\end{aligned}
$$

Thus

$$
\begin{aligned}
& \beta=\left(\frac{1}{n} \sum_{i=1}^{n}\left(x_{i}\right)^{\alpha}\right)^{1 / \alpha}, \\
& \alpha=\left[\frac{\sum_{i=1}^{n}\left(x_{i}\right)^{\alpha} \ln x_{i}}{\sum_{i=1}^{n}\left(x_{i}\right)^{\alpha}}-\frac{1}{n} \sum_{i=1}^{n} \ln x_{i}\right]^{-1} .
\end{aligned}
$$

2.2.3. Method of Moments. The $k$ th moment of the Weibull distribution, $\mu_{k}$, is defined by

$$
\mu_{k}=\beta^{k} \Gamma\left(1+\frac{k}{\alpha}\right)
$$

where $\Gamma(t)$ is a gamma function which is given by

$$
\Gamma(s)=\int_{0}^{\infty} e^{-x} x^{s-1} d x, \quad s>0 .
$$


TABLE 1: Claims size $x_{i}$ (millions Baht).

\begin{tabular}{|c|c|c|c|c|c|c|}
\hline 15.50 & 6.70 & 49.90 & 6.40 & 12.40 & 102.70 & 44.90 \\
\hline 56.50 & 138.90 & 107.30 & 13.20 & 13.10 & 37.70 & 5.70 \\
\hline 40.00 & 1.80 & 40.20 & 84.30 & 47.30 & 112.20 & 9.20 \\
\hline 28.50 & 0.90 & 43.10 & 3.60 & 45.80 & 70.00 & 2.30 \\
\hline 35.30 & 7.20 & 64.60 & 13.00 & 2.40 & 1.40 & 2.10 \\
\hline 7.50 & 31.50 & 4.20 & 37.20 & 0.70 & 24.40 & 14.20 \\
\hline 20.10 & 0.40 & 33.20 & 9.30 & 10.80 & & \\
\hline
\end{tabular}

TABLE 2: Shape and scale parameters for a variety of estimation methods.

\begin{tabular}{|c|c|c|c|c|}
\hline Method & Type & $F_{i}$ & $\alpha$ & $\beta$ \\
\hline 1 & Least squares method 1 & 5.1 & 0.8405 & 28.7721 \\
\hline 2 & Least squares method 2 & 5.2 & 0.8310 & 28.8602 \\
\hline 3 & Least squares method 3 & 5.3 & 0.7984 & 29.1888 \\
\hline 4 & Least squares method 4 & 5.4 & 0.8580 & 28.6168 \\
\hline 5 & Maximum likelihood estimation & - & 0.8633 & 28.8668 \\
\hline 6 & Method of moments & - & 0.9286 & 30.0055 \\
\hline
\end{tabular}

Setting $k=1$, we obtain

$$
\mu=\beta \Gamma\left(1+\frac{1}{\alpha}\right)
$$

Since $\sigma^{2}=\mu_{2}-\mu^{2}$,

$$
\sigma^{2}=\beta^{2} \Gamma\left(1+\frac{2}{\alpha}\right)-\beta^{2}\left(\Gamma\left(1+\frac{1}{\alpha}\right)\right)^{2} .
$$

We calculate a coefficient of variation $(\mathrm{CV})$ of the Weibull distribution from the formula

$$
C V=\frac{\sigma}{\mu}=\frac{\sqrt{\beta^{2} \Gamma(1+2 / \alpha)-\beta^{2}(\Gamma(1+1 / \alpha))^{2}}}{\beta \Gamma(1+1 / \alpha)} .
$$

If we apply the bisection method to (27), we get the parameter $\alpha$. Thereafter, we substitute $\alpha$ in (25) which obtains the parameter $\beta$.

2.2.4. Kolmogorov-Simirnov Test. The Kolmogorov-Simirnov (KS) test is a distance test. The KS test works well with small samples. Let $y_{1}<y_{2}<\cdots<y_{n}$ be the order of statistics of a random sample $x_{1}, x_{2}, \ldots, x_{n}$. The empirical distribution function is of the form

$$
F_{n}(x)= \begin{cases}0 & \text { if } x<y \\ \frac{k}{n} & \text { if } y_{k} \leq x \leq y_{k+1}, k=1,2, \ldots, n-1, \\ 1 & \text { if } y_{n} \leq x\end{cases}
$$

Let $F_{0}(x)$ be a hypothesized distribution function, so the KS statistic is defined by $D_{n}=\sup _{x}\left\{\left|F_{n}(x)-F_{0}(x)\right|\right\}$.

Throughout this research, we use the data of the cost of all claim sizes that are greater than twenty million Baht from the fire insurance of Thai Reinsurance Public. The amount $x_{i}$ is shown in Table 1 which is greater than twenty million
Baht, i.e., $x_{i}=y_{i}-20$. Boonta et al. [5] estimated the shape parameters $\alpha$ and scale parameters $\beta$ from a variety of estimation methods by means of a minimum Chi-squared test as shown in Table 2 and using the data according to Table 1 .

We perform the KS test according to the six methods as shown in Table 2 . The hypothesis $H_{0}$ : the set data in Table 1 assumed the Weibull distribution with parameters $\alpha$ and $\beta$. The result shows that the set of data cannot reject the Weibull distribution at $95 \%$ confidence. For example, the hypothesis $\mathrm{H}_{0}$ : the set of data assumed the Weibull distribution with $\alpha=0.8633$ and $\beta=28.8668$. The $\mathrm{KS}$ critical value at $95 \%$ confidence is $1.36 / \sqrt{47}=0.1984$ whereas the KS statistic value is 0.0709 . Thus the set of data accepts the Weibull distribution at $95 \%$ confidence. Figure 1 shows a plot of the cumulative empirical distribution function $F_{n}(x)$ with the cumulative Weibull distribution function $F_{0}(x)$.

\section{Results}

3.1. Part I: The Direct Search Technique. The direct search technique is a numerical optimization method. The principle of the direct search technique is iterative and random shifting from the beginning solution to a better solution. The direct search technique process can be described as follows.

Step 1. Begin from the simple initial parameters $\alpha$ and $\beta$, i.e., $\alpha=0.5, \beta=31.06$ ( $\beta$ is mean of 47 observe data) and compute the KS statistic value for $\alpha$ and $\beta$.

Step 2. Calculate value $\alpha$ to $\alpha^{\prime}$ and $\beta$ to $\beta^{\prime}$ by using the formulas:

$$
\begin{array}{r}
\alpha^{\prime}=\alpha+0.01(0.5-u) \\
\text { and } \beta^{\prime}=\beta+0.1(0.5-u)
\end{array}
$$

where $u$ is randomly chosen from $[0,1]$. 


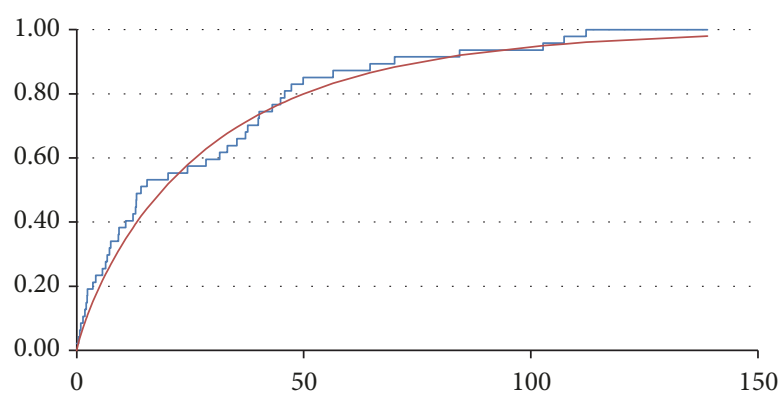

Figure 1: The empirical CDF $F_{n}(x)$ (blue color) and the Weilbull CDF $F_{0}(x)$ (red color).

TABLe 3: Parameters $\alpha, \beta$, and the KS statistic value by the direct search technique (Initial parameters $\alpha=0.5, \beta=\bar{x}=31.06$ ).

\begin{tabular}{lccc}
\hline Times & $\alpha$ & $\beta$ & Value for the KS test \\
\hline 1 & 0.5049 & 31.0142 & 0.1262 \\
10 & 0.5489 & 30.6153 & 0.1088 \\
100 & 0.7652 & 29.5447 & 0.0544 \\
1000 & 0.7652 & 29.5451 & 0.0544 \\
\hline
\end{tabular}

Step 3. Calculate the KS statistic value by using $\alpha^{\prime}$ and $\beta^{\prime}$.

Step 4. Compare the KS value of Step 1 and Step 3.

(4.1) If the KS statistic value of Step 1 is less than Step 3, then we do Step 2 again.

(4.2) If (4.1) is false we set $\alpha=\alpha^{\prime}, \beta=\beta^{\prime}$ and then we perform Step 2 again.

Step 5. Iterate until the process is complete (four-decimal place accuracy).

If value for the KS test is approximated to four-decimal place accuracy, then the direct search technique is completed. We perform the simulation 1000 times. The results are shown in Table 3.

Table 4 shows that the value for the KS test of the direct search technique equals 0.0544 which is less than that of the other methods. Therefore, the direct search technique with given simple initial parameters $\alpha=0.5$ and $\beta=$ $\bar{x}=31.06$ affect the preciseness of the parameter estimation (four-digit accuracy) more than the least squares method, the maximum likelihood estimation, and the method of moments. Consequently, the set data in Table 1 accepts the Weibull distribution at 95\% confidence with parameters $\alpha=$ 0.7526 and $\beta=29.5450$. These parameters will be used to approximate the minimum initial capital of the surplus process (12) which will be explained in Part II.

\subsection{Part II: The Simulation of the Ruin Probability and the Minimum Initial Capital}

3.2.1. The Simulation of the Ruin Probability. We consider the surplus process (12) under the condition of the claim time $T_{i}$ for $i=1,2, \ldots, n$ with $0 \leq T_{1} \leq \cdots \leq T_{n} \leq \widetilde{T}$ when $\widetilde{T}=$
365 days. A simulation method is used to compute the ruin probability $P\left(\left\{U_{n}<0 \exists n \in \mathbb{N}\right\}, \sum_{i=1}^{n} Z_{i} \leq 365 \mid U_{0}=u\right)$, where $Z_{i}=T_{i}-T_{i-1}$. The flowchart is shown in Figure 2 .

In Figure 2, the premium rate for one unit of time, $c$, can be calculated by the expected value principle, i.e.,

$$
c=(1+\theta) \frac{E Y_{1}}{E Z_{1}}
$$

where $\left\{Y_{1}, Y_{2}, \ldots, Y_{n}\right\}$ is the sequence of claims which are assumed to be i.i.d. and $\left\{Z_{1}, Z_{2}, \ldots, Z_{n}\right\}$ is the sequence of the Poisson random variable which is also assumed to be i.i.d. We estimate that the parameter $\lambda$ is equal to 7.766. Then we get the premium rate as the following equation:

$$
\begin{aligned}
c & =(1+\theta) \frac{E Y_{1}}{E Z_{1}}=(1+\theta) \frac{(29.5450) \Gamma(1+1 / 0.7652)}{7.766} \\
& =(1+\theta)(4.4570)
\end{aligned}
$$

Thereafter we perform the simulation of the ruin probability by setting the initial capital $u=570,600$, $630,660,690 \ldots, 1470$ million Baht. The simulations are computed 10,000 iterations (see Figure 2). The results are shown in Figure 3.

3.2.2. Minimum Initial Capital. We consider the relationship between the ruin probability $\Phi(u, 365):=y$ and the initial capital $u$ in Figure 3 which is the quadratic function,

$$
y=a u^{2}+b u+c
$$

Next, we use the quadratic regression method in (32); we obtain the parameters $a, b$ and $c$ as shown in Table 5 .

Finally, we consider that the ruin probability is not greater than $\alpha$, i.e.,

$$
\begin{aligned}
\Phi(u, 365) & \leq \alpha \\
a u^{2}+b u+c & \leq \alpha \\
a u^{2}+b u+c-\alpha & \leq 0
\end{aligned}
$$




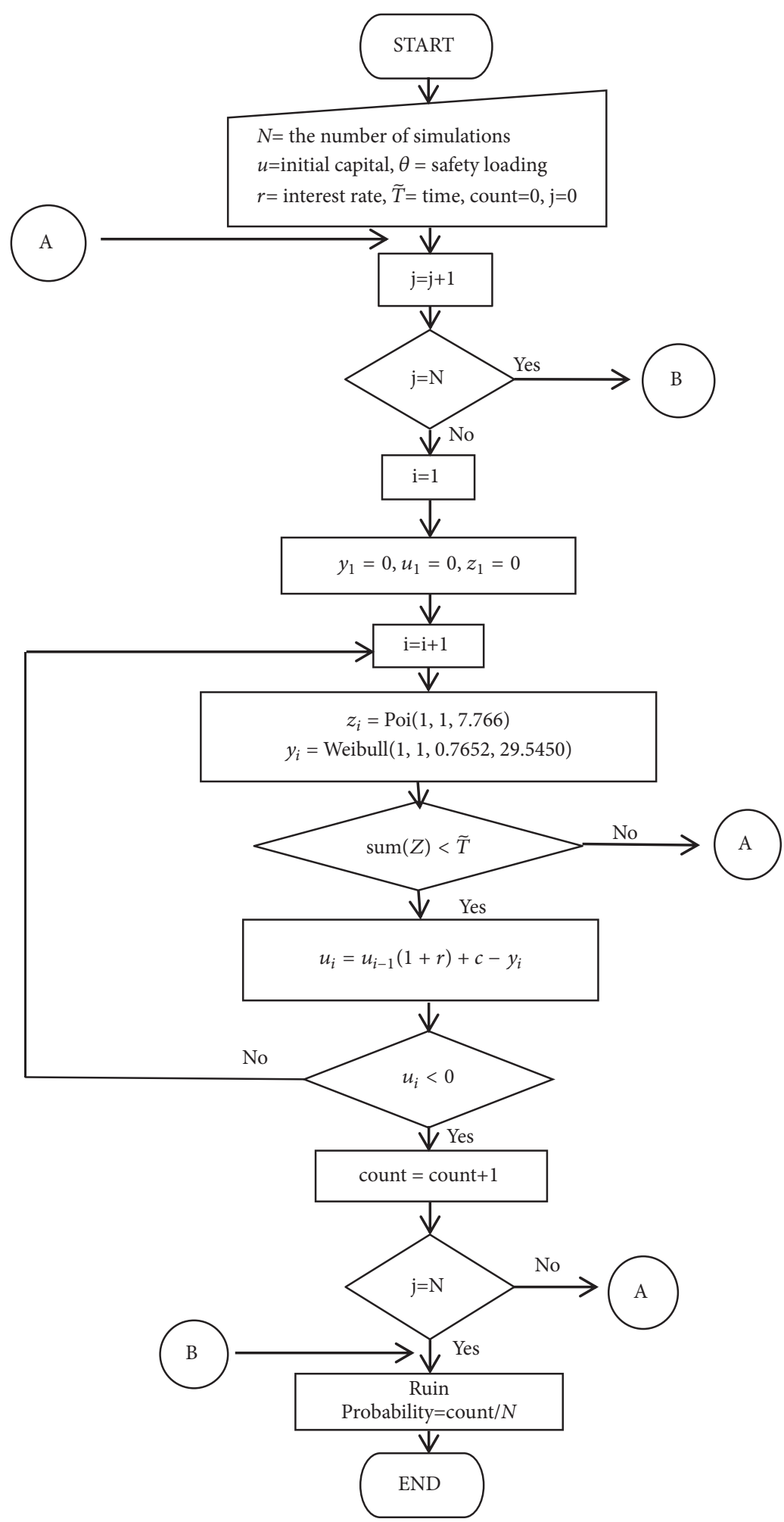

FIGURE 2: The flow chart of a simulation of the ruin probability.

For example, we can apply (33) to calculate the MIC in the case of $\theta=0.1$, and $\alpha=0.01$. Consequently, we obtain

$$
\begin{gathered}
\left(-9.9562330409 \times 10^{-7}\right) x^{2}+(0.001282542) x \\
+(0.589108899)-0.01 \leq 0 .
\end{gathered}
$$

and we get $x \in(-\infty,-354.16184) \cup(1642.3413, \infty)$. Therefore, the MIC of a reinsurance company has to hold for ensuring that the ruin probability is not greater than $\alpha=0.01$, i.e., the MIC is equal to 1642.3413 million Baht. In the other cases of $\theta$ and $\alpha$, we use a similar method. Then, we obtain the MIC shown in the Table 6. 


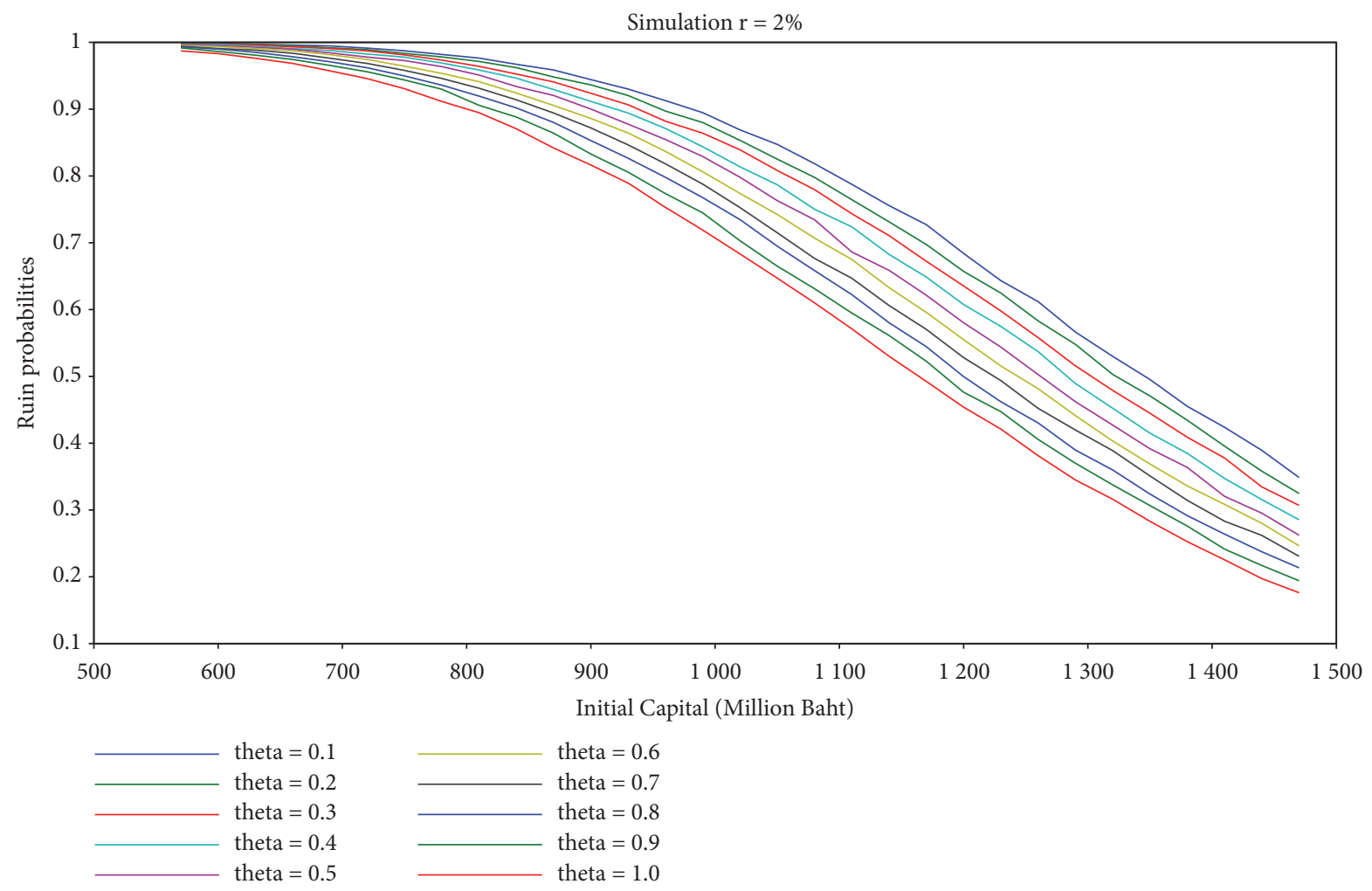

FIGURE 3: The relation between the initial capital and the ruin probability in the case of $r=2 \%$.

TABLE 4: The value for the KS test from a variety of estimation methods.

\begin{tabular}{lcccc}
\hline Method & Type & $\alpha$ & $\beta$ & Value for the KS test \\
\hline 1 & Least squares method 1 & 0.8405 & 28.7721 & 0.0649 \\
2 & Least squares method 2 & 0.8310 & 28.8602 & 0.0636 \\
3 & Least squares method 3 & 0.7984 & 29.1888 & 0.0591 \\
4 & Least squares method 4 & 0.8580 & 28.6168 & 0.0674 \\
5 & Maximum likelihood estimation & 0.8633 & 28.8668 & 0.0709 \\
6 & Method of moments & 0.9286 & 30.0055 & 0.0964 \\
7 & Direct search technique & 0.7652 & 29.5450 & 0.0544 \\
\hline
\end{tabular}

TABLE 5: The parameters $a, b$, and $c$ of the quadratic function by varying safety loading $\theta$.

\begin{tabular}{lccc}
\hline$\theta$ & $a$ & $b$ & $c$ \\
\hline 0.1 & $-9.9562330409 \times 10^{-7}$ & 0.001282542 & 0.589108899 \\
0.2 & $-9.7285348409 \times 10^{-7}$ & 0.001200587 & 0.632291576 \\
0.3 & $-9.3646878406 \times 10^{-7}$ & 0.00109436 & 0.685620907 \\
0.4 & $-8.9480810258 \times 10^{-7}$ & 0.000975717 & 0.744250813 \\
0.5 & $-8.4790507621 \times 10^{-7}$ & 0.000846694 & 0.80705413 \\
0.6 & $-7.8222126156 \times 10^{-7}$ & 0.00068849 & 0.879791861 \\
0.7 & $-7.1361178877 \times 10^{-7}$ & 0.000523748 & 0.955127497 \\
0.8 & $-6.5784995253 \times 10^{-7}$ & 0.000382004 & 1.020181037 \\
0.9 & $-5.8276047039 \times 10^{-7}$ & 0.000207764 & 1.097419007 \\
1.0 & $-5.1967843269 \times 10^{-7}$ & 0.000059240 & 1.160626457 \\
\hline
\end{tabular}


TABLE 6: The MIC of a reinsurance company has to hold for ensuring that the ruin probability is not greater than $\alpha$.

\begin{tabular}{lccc}
\hline$\theta$ & $\begin{array}{c}\text { Premium rate } \\
c=(1+\theta)(4.4570)\end{array}$ & $\begin{array}{c}\text { MIC million Baht } \\
(\alpha=0.01)\end{array}$ & $\begin{array}{c}\text { MIC million Baht } \\
(\alpha=0.05)\end{array}$ \\
\hline 0.1 & 4.9027 & 1642.3413 & 1622.0112 \\
0.2 & 5.3484 & 1627.1924 & 1606.6316 \\
0.3 & 5.7941 & 1615.2543 & 1594.3263 \\
0.4 & 6.2398 & 1602.4812 & 1581.1251 \\
0.5 & 6.6855 & 1589.8425 & 1567.9948 \\
0.6 & 7.1312 & 1582.7270 & 1560.1270 \\
0.7 & 7.5769 & 1574.8999 & 1551.4706 \\
0.8 & 8.0226 & 1563.0873 & 1538.9719 \\
0.9 & 8.4683 & 1555.8489 & 1530.7069 \\
1.0 & 8.9140 & 1546.0777 & 1520.0044 \\
\hline
\end{tabular}

Table 6 shows that if $\theta$ increases, it affects the premiums which also increase and then the MIC decreases under given $\alpha$-regulation.

\section{Conclusion and Suggestion}

This work presents the use of the direct search technique to estimate the parameters of a Weibull distribution. The direct search technique is compared with the least squares method, the maximum likelihood estimation, and the method of moments. The results show that the direct search technique is more accurate than the other methods (accuracy of four decimal points). Fire accident data is used for special cases exceeding 20 million Baht. The results show that if the safety loading is increased, the MIC decreases under the given $\alpha$-regulation; i.e., reinsurance will enable the financial management plan to prevent insolvency.

\section{Data Availability}

The data used to support the findings of this study are included within the article.

\section{Conflicts of Interest}

The authors declare that there are no conflicts of interest regarding the publication of this paper.

\section{Acknowledgments}

This paper is supported by the Faculty of Science and Engineering, Kasetsart University, Chalermphrakiat Sakon Nakhon Province Campus, and Mathematics and Statistics Program, Faculty of Science and Technology, Sakon Nakhon Rajabhat University, Sakon Nakhon, 47000, Thailand.

\section{References}

[1] B. Bergman, "On the estimation of the Weibull modulus," Journal of Materials Science Letters, vol. 3, no. 8, pp. 689-692, 1984.
[2] J. D. Sullivan and P. H. Lauzon, "Experimental probability estimators for Weibull plots," Journal of Materials Science Letters, vol. 5, no. 12, pp. 1245-1247, 1986.

[3] A. Khalili and K. Kromp, "Statistical properties of Weibull estimators," Journal of Materials Science, vol. 26, no. 24, pp. 6741-6752, 1991.

[4] K. Trustrum and A. D. S. Jayatilaka, "On estimating the Weibull modulus for a brittle material," Journal of Materials Science, vol. 14, no. 5, pp. 1080-1084, 1979.

[5] S. Boonta, A. Sattayatham, and P. Sattayatham, "Estimation of Weibull parameters using a randomized neighborhood search for the severity of fire accidents," Journal of Mathematics and Statistics, vol. 9, no. 1, pp. 12-17, 2013.

[6] F. Lundberg, Approximerad Framställning av Sannolikhetsfunktionen, II. Atterförsäkering av Kollektivrisker, Almqvist \& Wiksell, Uppsala, 1903.

[7] K. P. Pavlova and G. E. Willmot, "The discrete stationary renewal risk model and the gerber - shui discounted penalty function," Insurance: Mathematics \& Economics, vol. 35, no. 2, pp. 267-277, 2004.

[8] D. C. M. Dickson, Insurance Risk and Ruin, Cambridge University Press, New York, NY, USA, 2005.

[9] S. Li, "Distributions of the surplus before ruin, the deficit at ruin and the claim causing ruin in a class of direct time renewal risk models," Scandinavian Actuarial Journal, vol. 4, pp. 271-284, 2005.

[10] S. Li, "On a class of direct time renewal risk models," Scandinavian Actuarial Journal, vol. 4, pp. 241-260, 2005.

[11] R. Wang and H. Liu, "On the ruin probability under a class of risk process," The Journal of the International Actuarial Association, vol. 32, no. 1, pp. 81-90, 2002.

[12] W.-S. Chan and L. Zhang, "Direct derivation of finite-time ruin probabilities in the discrete risk model with exponential or geometric claims," North American Actuarial Journal, vol. 10, no. 4, pp. 269-279, 2006.

[13] P. Sattayatham, K. Sangaroon, and W. Klongdee, "Ruin probability-based initial capital of the discrete-time surplus process," Variance: Advancing the Science of Risk, vol. 7, pp. 7481, 2013.

[14] C. Weng, Y. Zhang, and K. S. Tan, "Ruin probabilities in a discrete time risk model with dependent risks of heavy tail," Scandinavian Actuarial Journal, no. 3, pp. 205-218, 2009.

[15] A. Luesamai and S. Chongcharoen, "Upper bound of ruin probability for an insurance discrete-time risk model with 
proportional reinsurance and investment," Pakistan Journal of Statistics and Operation Research, vol. 14, no. 3, pp. 595-614, 2018.

[16] M. Bourguignon, B. S. Rodrigo, and M. C. Gauss, "The Weibull - G family of probability distributions," Journal of Data Science, vol. 12, pp. 53-68, 2014.

[17] K. E. Aifantis, A. Konstantinidis, and S. Forest, "Modeling strain localization bands in metal foams," Journal of Computational and Theoretical Nanoscience, vol. 7, no. 2, pp. 1-7, 2010. 


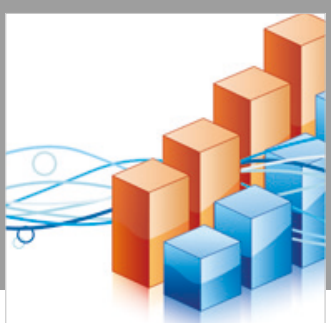

Advances in

Operations Research

\section{-n-m}
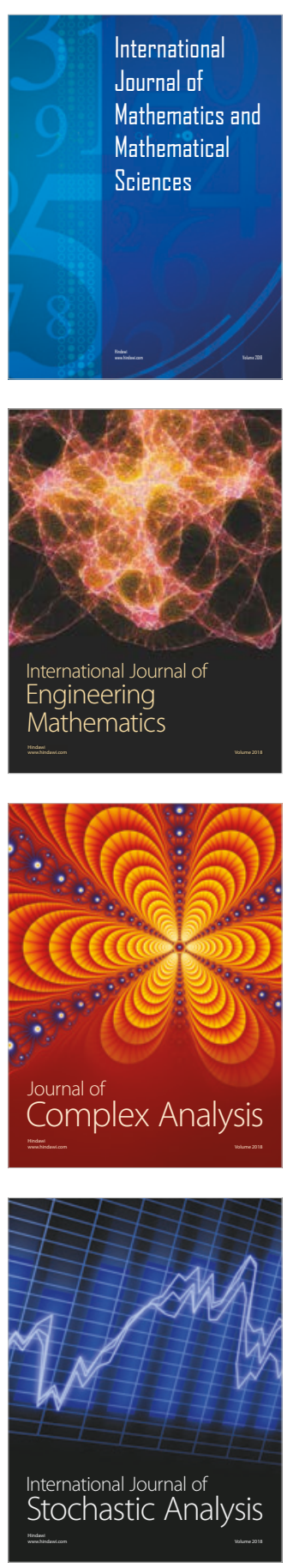
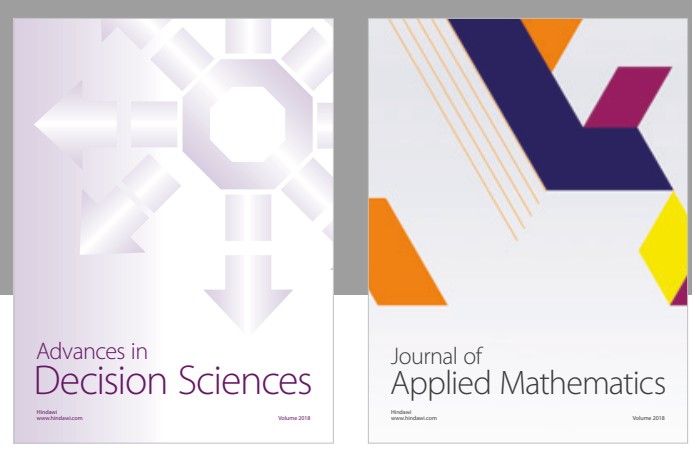

Journal of

Applied Mathematics
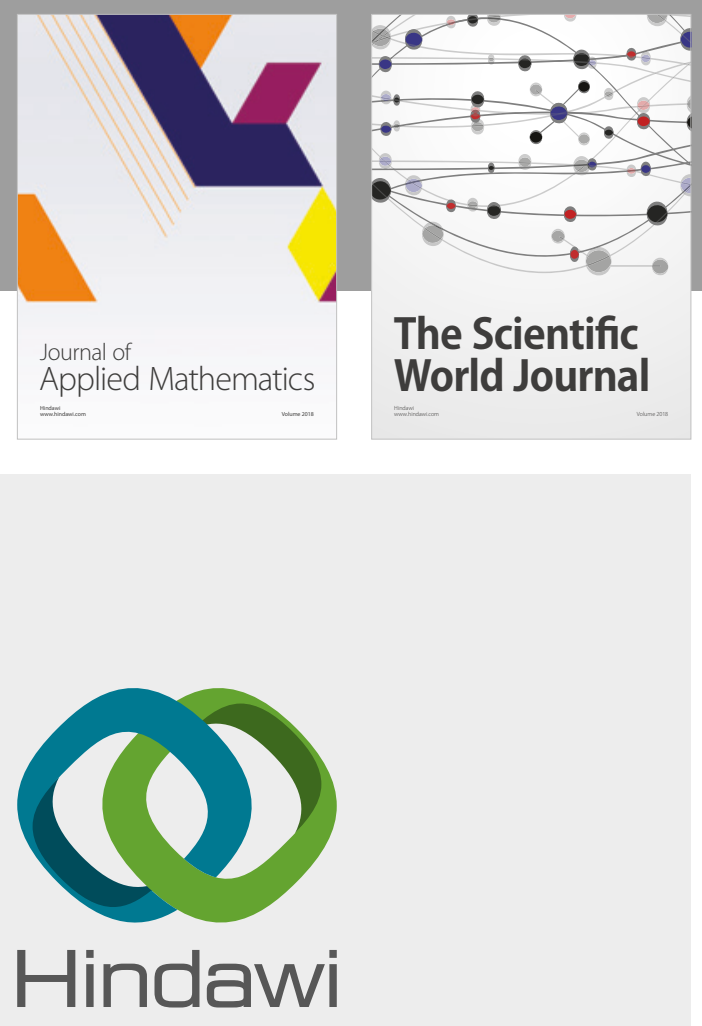

Submit your manuscripts at

www.hindawi.com

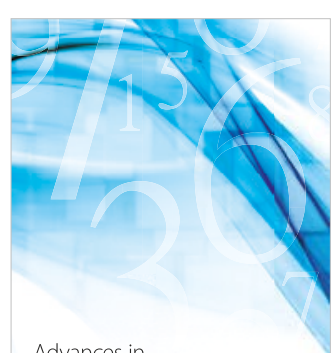

Advances in
Numerical Analysis
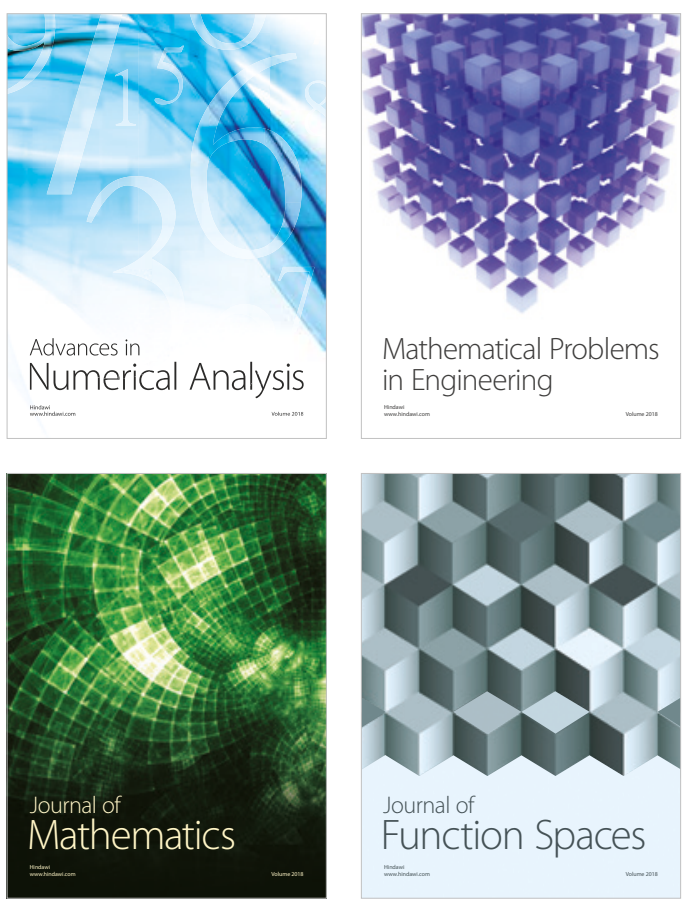

Mathematical Problems in Engineering

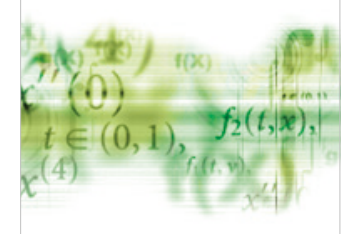

International Journal of

Differential Equations

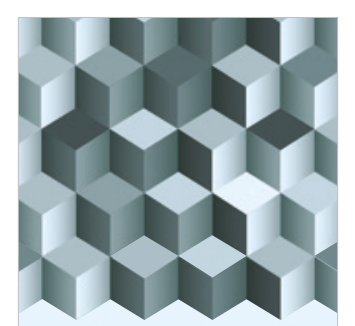

Journal of

Function Spaces

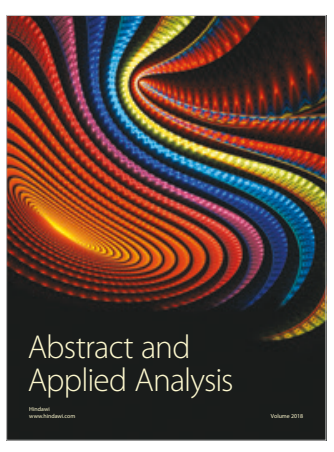

The Scientific

World Journal

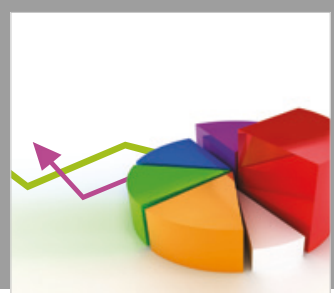

Journal of

Probability and Statistics
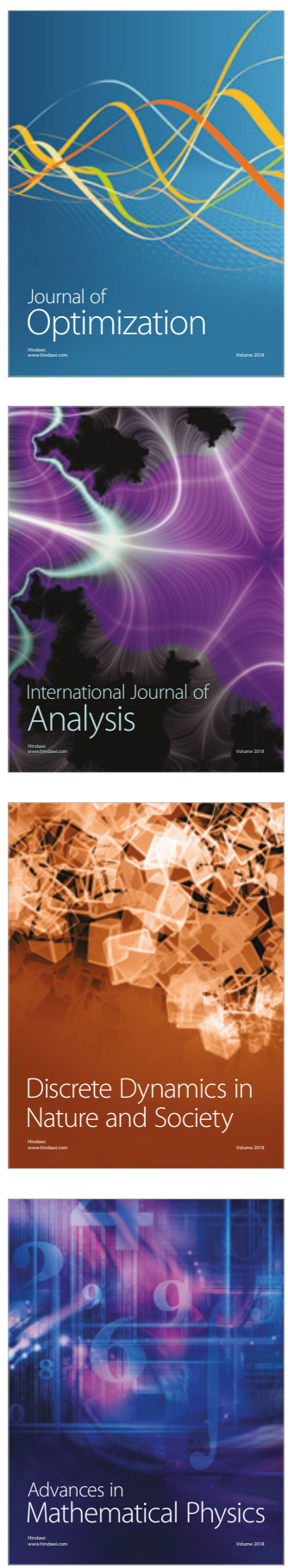